\title{
How to know what works in alleviating poverty: Learning from experimental approaches in qualitative research
}

\author{
Dr. Alexandra Hartman \\ The School of Public Policy \\ University College London \\ 29-30 Tavistock Square \\ WC1H 9QU London UK \\ +44 (0)20 76794977 \\ alexandra.hartman@ucl.ac.uk
}

\author{
Dr. Florian G. Kern \\ Department of Government \\ University of Essex \\ Wivenhoe Park \\ CO4 3SQ Colchester UK \\ +44 (0)1206 872745 \\ fkern@essex.ac.uk
}

\begin{abstract}
Experimental studies of poverty alleviation have stimulated an interdisciplinary discussion on what constitutes robust evidence to inform policy and benefit the poor. These studies emphasize research transparency and reporting standards, pre-registration, data sharing, replication and aggregated evidence. Though imperfect, such practices help to identify what works under what conditions. We argue that researchers should also explore how similar practices could be tailored for qualitative research on the politics of poverty alleviation. We outline a research framework motivated by the experiment-focused Metaketa initiative that incorporates the strengths of qualitative inquiry. We present the eleven pillars of a qualitative Metaketa.
\end{abstract}

\section{Introduction: Experimental advances in transparency and evidence aggregation}

Advances in experimental studies of poverty and development have motivated an expansion of causal inference based work across the social sciences - including in political science, psychology, and economics - drawing on carefully designed interventions, as well as fine-grained individual, household and community data. Seminal contributions include Banerjee et al. (2007), Duflo (2001), Duflo, Kremer and Robinson (2011) or Miguel and Kremer (2004) (and many others). In addition to generating critical substantive knowledge, these advances have also reoriented methodological debates about the best ways to build knowledge and use research to inform solutions to policy problems. In particular, experimentalists have promoted the idea of transparent inference and pre-registration (Miguel et al., 2014) and aggregated and coordinated analysis across contexts (Banerjee et al., 2015) to produce robust evidence and to "know what works" in development policy. The debate on the value of data sharing, production transparency and systematic knowledge aggregation for quantitative research feeds into ongoing work by scholars who employ qualitative data. It also introduces debates about applying new reporting standards, partly inspired by experimentalists' responses to these issues (Christensen, Hartman and Samii, Registered October 17, 2017; Kern and Gleditsch, 2017; Kapiszewski and Karcher, 2019; Jacobs et al., 2019; Elman and Kapiszewski, 2014; Jacobs, Forthcoming). Contributing to this nascent debate, in the following, we explore one development in coordinated and aggregated experimental research, the Metaketa initiative, and its implications for emerging work that relies on qualitative research.

\section{A qualitative Metaketa}

The Metaketa initiative - started by the Evidence in Governance and Politics (EGAP) network - was "designed to encourage replication, increase harmonization between studies such that meaningful aggregation is possible, and foster commitment to design and reporting standards that guard against selective reporting and publication bias. Its overarching goal is to promote cumulative learning in the social sciences" (Dunning et al., 2019, 8). The Metaketa (a Basque word meaning 'accumulation') model proposes an approach to research with scholars and 
policy practitioners in close collaboration to find answers of major importance to development policy. Metaketas emphasize coordinated studies and integrated research designs to produce comparative and generalizable evidence in the social sciences. The four implemented Metaketa projects draw on the fact that practitioner organisations engaged in the development sector use similar programs globally to improve voter information, taxation compliance, policing, and natural resource usage. ${ }^{1}$ By experimentally randomizing these interventions, teams of scholars can work together to understand if and how common interventions achieve the goals they set out to obtain. Within projects, collaborators commit to data transparency, replication, and metaanalysis of all the data collected.

How could the Metaketa model be employed across a set of coordinated projects that draw on qualitative data collected through fieldwork across different settings? Together a group of scholars and practitioners collaborated and met in the spring of 2019 to develop a model for a qualitative Metaketa. ${ }^{2}$ By using the ten pillars of the experimental Metaketa framework (Dunning et al., 2019, 27) and its emphasis on cumulative learning as a starting point, the group discussed the strengths and weaknesses of qualitative and quantitative approaches and how transparency and reporting standards unfold in qualitative research. The collaboration produced eleven basic pillars supporting a future qualitative Metaketa application: ${ }^{3}$

Common conceptual framework. In order to carry to out integrated research, the researchers participating in the qualitative Metaketa should set out a common conceptual framework that generates a set of shared hypotheses that will be tested in each case included in the Metaketa. A co-generated and shared conceptual framework provides a basis for comparing evidence generated across different cases.

Common analytic framework. In order make the most from a shared conceptual framework, researchers should employ a common analytic framework across all the cases. While there are numerous frameworks that could yield useful results, process tracing "or the use of evidence from within a case to make inferences about causal explanations of that case" (Bennett and Checkel 2014) can be of particular value, because of its comparative advantage in using rich within-case data to identify causal processes and mechanisms.

Multiple studies in diverse contexts. Similar to previous and ongoing Metaketas, the goal of the qualitative Metaketa would be to produce comparable evidence across diverse study sites. As a result, the qualitative Metaketa is similarly committed to including at least four or more studies in different contexts within the Metaketa.

A common phenomenon under study. In order to include multiple studies in diverse contexts within one Metaketa, all the studies should focus on a common phenomenon or a set of phenomena prompting a common research question shared across all cases.

Pre-registered and harmonized protocols for data generation and inference. In order for researchers to engage with a common analytic framework, a set of harmonized protocols for data

\footnotetext{
${ }^{1}$ For more information on the four projects see https://egap.org/metaketa

${ }^{2}$ We thank the members of the group: Kristin Bakke, Anne Buffardi, Catherine Boone, Nic Cheeseman, Pilar Domingo, Ismene Gizelis, Emmanuel Gyimah-Boadi, Macartan Humphreys, Alan Jacobs, Milli Lake, Gabrielle Lynch, Winnie Mitullah, Lauren MacLean, Cyrus Samii, Alexandra Scacco, Mareike Schomerus, Anastasia Shesterinina and Jonathan Slapin.

${ }^{3}$ We regard these eleven pillars as the essential base for a qualitative Metaketa. Future applications may develop additional pillars.
} 
generation, including but not limited to interview guides, focus group discussion guides, archival research plans, and observational data gathering guides should be created by all the participating researchers prior to the start of data collection. In addition to shared data generation protocols, researchers should also agree to the same approaches to data analysis, i.e. harmonize before the data is collected how the produced information is to be analyzed (e.g. with computer assisted qualitative data analysis), and, crucially, what types of information would constitute evidence of a particular causal process that the research sets out to uncover. A first version of all protocols and analysis frameworks would be agreed upon prior to data collection (although iteration is not only possible, but encouraged) and - to the best extent possible - recorded in a qualitative pre-analysis plan that is time stamped and made accessible to the wider research community online (or gated until data analysis is finalised).

Iterative adjacent arms. One of the key features of much qualitative work - and particularly fieldwork - is iteration. Iteration, or the updating of the conceptual framework, data generation protocols, and data analysis frameworks during the research process, should be a key part of the qualitative Metaketa and is encouraged alongside or adjacent to work with the co-generated harmonized protocols. With a focus on documenting iteration, the qualitative Metaketa aims to show how different kinds of information are produced when following pre-determined protocols versus those that are shaped by iteration. Documented iteration should be encouraged not only within cases, but also as part of "cross-pollination" across cases within the Metaketa, so that collaborators can learn from insights from other cases to update their own analysis dynamically, e.g. by exploring an inductively generated hypothesis from one case deductively across cases. The goal should be to explore how iteration contributes to research and how it may help avoid key pitfalls, such as confirmation bias.

Scope conditions. Much research explores scope conditions, or the pre-existing factors or variables which are necessary for a particular process or causal chain to unfold in a specific way. The qualitative Metaketa puts understanding these moderating factors at the forefront of the project such that researchers are focused not only on identifying if specific causal mechanisms exist at all, but also how and why certain subgroups or contexts are necessary for the mechanisms' explanatory power.

Meta analysis. The goal of the qualitative Metaketa should be to produce research that informs both a rich understanding of a phenomenon in a particular case as well as a meta-analysis of that phenomenon across all cases. Participating researchers therefore should commit to participating in a meta analysis involving data from all the cases pooled together.

Study researcher effects. Much published research in mainstream political science shies away from engaging with a fundamental question at the heart of both data generation and data analysis: the impact of both a socio-political context and a given researcher's position within that context on the knowledge that is generated during research. While differing interpretations are often leveraged by multi-member research teams to great effect, this work is often hidden or under explored. The qualitative Metaketa takes advantage of the fact that multiple researchers work on each case and should provide specific mechanisms for documenting how different researchers gather and analyze the same data within one case. In particular, the qualitative Metaketa aims to explore this dynamic across researchers with "insider" identities closely linked with the research site and those with "outsider" identities who are visitors or guests and how these different positions generate different kinds knowledge. Moreover, collaborators working on different cases within the qualitative Metaketa should analyze each other's data 
before knowing the other's findings to validate conclusions and identify critical areas of differing inference.

Ethics. Given the intense human interaction often created during the qualitative research process, the qualitative Metaketa commits to putting ethics at the centre of its project. This includes both a deep engagement with how to conduct ethical research and also an acknowledgement that the very human component of qualitative research frequently means that research does change participants' lives in diverse and unintended ways (Cronin-Furman and Lake, 2018).

Policy relevance. Like the quantitative Metaketa, the qualitative Metaketa is committed to focusing on research questions at the intersection of theory of policy that are relevant to audiences inside and outside of the academic sphere.

\section{Conclusion: Learning from Experimental Approaches in Qualitative Research}

We propose the eleven pillars of the qualitative Metaketa as a way for researchers to explore how evidence aggregation and applied transparency can benefit qualitative research on poverty alleviation. Qualitative research is uniquely designed to provide insights into the processes at play explaining poverty and development. While experimentalists have been at the forefront of promoting reporting standards and systematic knowledge aggregation, a qualitative Metaketa could emphasize the strengths of iterative and context-specific work, a critical complement to existing research on what works in alleviating poverty.

\section{References}

Banerjee, Abhijit, Esther Duflo, Nathanael Goldberg, Dean Karlan, Robert Osei, William Parienté, Jeremy Shapiro, Bram Thuysbaert and Christopher Udry. 2015. "A multifaceted program causes lasting progress for the very poor: Evidence from six countries." Science 348(6236):1260799-1-16.

Banerjee, Abhijit V, Shawn Cole, Esther Duflo and Leigh Linden. 2007. "Remedying education: Evidence from two randomized experiments in India." The Quarterly Journal of Economics 122(3):1235-1264.

Bennett, A., \& Checkel, J., T. (Eds.). (2014). Process tracing: From metaphor to analytic tool. Cambridge, UK: Cambridge University Press.

Christensen, Darin, Alexandra Hartman and Cyrus Samii. Registered October 17, 2017. "Legibility and External Investment: An Institutional Natural Experiment in Liberia." Qualitative Preanalysis Plan, Open Society Foundation.

Cronin-Furman, Kate and Milli Lake. 2018. "Ethics Abroad: Fieldwork in Fragile and Violent Contexts." PS: Political Science \& Politics 51(3):607-614.

Duflo, Esther. 2001. "Schooling and labor market consequences of school construction in Indonesia: Evidence from an unusual policy experiment." American economic review 91(4):795-813.

Duflo, Esther, Michael Kremer and Jonathan Robinson. 2011. "Nudging farmers to use fertilizer: Theory and experimental evidence from Kenya." American economic review 101(6):2350-90. 
Dunning, Thad, Guy Grossman, Macartan Humphreys, Susan D Hyde, Craig McIntosh and Gareth Nellis. 2019. Information, Accountability, and Cumulative Learning: Lessons from Metaketa I. Cambridge University Press.

Elman, Colin and Diana Kapiszewski. 2014. "Sharing qualitative data: the launch of the Qualitative Data Repository." The Washington Post: Monkey Cage (February 25, 2014). URL: https://wapo.st/1hlKiYm?tid=ss_tw\&utm_term=.394f37714736

Jacobs, Alan. Forthcoming. The Production of Knowledge: Enhancing Progress in Social Science. New York, NY: Cambridge University Press. chapter Pre-Registration and Results-Free Review in Observational and Qualitative Research.

Jacobs, Alan M, Tim Büthe, Ana M Arjona, Leonardo R Arriola, Eva Bellin, Andrew Bennett, Lisa Björkman, Erik Bleich, Zachary Elkins, Tasha Fairfield et al. 2019. "Transparency in Qualitative Research: An Overview of Key Findings and Implications of the Deliberations." American Political Science Association Organized Section for Qualitative and Multi-Method Research, Qualitative Transparency Deliberations, Working Group Final Reports (July 2019).

. URL: https://ssrn.com/abstract $=3430025$

Kapiszewski, Diana and Sebastian Karcher. 2019. "Transparency in Practice in Qualitative Research.” APSA Pre-Prints. URL:

https://preprints.apsanet.org/engage/apsa/article-details/5d94c7762f41c7001256af6d

Kern, Florian G and Kristian Skrede Gleditsch. 2017. "Exploring Pre-Registration and PreAnalysis Plans for Qualitative Inference.” Preprint pp. 1-15.

Miguel, Edward, Colin Camerer, Katherine Casey, Joshua Cohen, Kevin M Esterling, Alan Gerber, Rachel Glennerster, Don P Green, Macartan Humphreys, Guido Imbens et al. 2014. "Promoting transparency in social science research." Science 343(6166):30-31.

Miguel, Edward and Michael Kremer. 2004. "Worms: identifying impacts on education and health in the presence of treatment externalities." Econometrica 72(1):159-217. 